\title{
New MoM Code Incorporating Multiple Domain Basis Functions
}

\author{
Albert A. Lysko ${ }^{1}$ \\ ${ }^{1}$ Council for Scientific and Industrial Research (CSIR): Meraka Institute, PO Box 395, Pretoria 0001, South \\ Africa, Tel.: +27 12841 4609, Fax:+27 12841 4720, Email: alysko@csir.co.za
}

\begin{abstract}
A new method of moments (MoM) code is discussed. The code supports standard functionality, e.g. fully automatic re-meshing and variable's assignment, ground plane and symmetry planes. In addition, the code realizes several new features. These include significant acceleration of the filling-in of the impedance matrix for high accuracy calculations on structures with redundancies, as well as realization of the multiple domain basis functions with a support for various aggregating basis function's profiles, including piecewise linear and piecewise sinusoidal. New profiles may be easily defined. The current realization of the code has been tested on several references and showed an excellent match to the reference results.
\end{abstract}

\section{Introduction}

The method of moments (MoM) $[1,2]$ is a popular method of solving electromagnetic problems. There are many codes available both commercially and freely [3]. Examples of such codes include FEKO, WIPL-D, PCAAD, SuperNEC and NEC-2. Most of the codes rely on the sub-domain piecewise linear (PWL) or piecewise sinusoidal (PWS) approximation for the current distribution [1]. WIPL-D [4], being an exception, uses higher order polynomial basis functions [2]. Most of the commercial codes are well optimized but are kept general to support financial sustainability for their providers. A particular problem is that the run times for specialized structures, including curved or corrugated elements, may be unnecessarily long due to the inability of the codes to support such structures in an efficient manner. There are also many highly specialized numerical electromagnetic codes [3], yet these codes are usually unable to handle general three-dimensional structures, as they were designed to handle very specific problems. The proposed code attempts to combine the ability to handle general structures with the ability to handle curved structures efficiently.

The number of arithmetic operations required to solve a system of linear algebraic equations resulting from the MoM is generally proportional to the third power of the number of unknowns [2]. Thus, minimization of the number of unknowns is a crucial component in making the method work fast. The speed and efficiency of the modeling heavily depends on the basis functions used. Higher order basis functions (HOBF), for example, are more efficient in modeling as one needs fewer unknowns per unit of length [2, 5]. A limitation for most of the existing codes including HOBF-based, is that they model smoothly curved structures using piecewise linear approximation of geometry. This often leads to an unnecessarily great number of unknowns used to model relatively small loop and spiral antennas, coils and other curved structures. This is because the program creates a dense mesh to describe the curvatures sufficiently accurately and assigns each mesh element at least one unknown, whilst the current may vary very slowly. The code described in this paper addresses this issue by applying macro-type $[13,14]$ basis functions called multiple domain basis functions (MDBF). The MDBFs are based on the original research [6-9].

Many structures include a substantial degree of symmetry, including hidden symmetries, or repetitions, which are not always easy to take advantage of in the code. Also, it is sometimes necessary to run computations with a high accuracy, leading to long run times. Most of the commercial codes try to address the former by using an advanced, usually hierarchical way to store the information about the geometry of the problem. This information is later used in the process of computing impedance matrix elements to avoid repeating redundant computations. However, there is another way to address this challenge by using memoization [10], a technique aiming to store and reuse the previously computed functions [11]. This technique has been realized in the code.

The paper is organized as follows. Section 2 gives an introduction into several novel methods realized in the code. Next, Section 3 provides a short description of the main functionalities supported by the code. Section 4 incorporates conclusions and next steps for the development. 


\section{Brief Overview of Some of the Novel Methods Used in the Code}

The multiple domain basis functions (MDBF) belong to the class of macro basis functions [13, 14] and can aggregate several original/old sub-domain basis functions into a single new composite basis function [6-8]. The new basis function is represented as a linear combination of the old basis functions with pre-defined constant coefficients. An aggregation of the old basis functions permits to unlink the mesh density from the number of unknowns $N$. This reduces the rank of the new impedance matrix, which is now based on the new set of MDBFs. The whole process may be expressed as a series of matrix multiplications $[6,15]$ and can result in up to $O\left(N^{3 / 4}\right)$ gain in speed compared to the traditional method of moments [15]. Also, in order to support the process of aggregation in the code automatically, three different re-meshing techniques have been devised [6]. The method can be easily fitted to most MoM codes, as shown in Figure 1 [6].

The calculation of the radiation pattern was also speeded up using an original method described in [17]. The method uses two schemes: Maclaurin series approximation and integration by parts, combined to achieve an optimum compromise between the speed and accuracy in computing radiation integrals. The technique is capable of handling the hierarchical polynomial basis functions of [2].

Another novel method implemented in the code is a memoization technique [10]. The term memoization is derived from Computer Science and refers to the use of cache to avoid repeated computation of functions. The technique was used to accelerate computation of the elements of the impedance matrix and showed acceleration factor exceeding an order of magnitude, subject to a high accuracy requirement.

\section{On Code Functionality and Application Results}

The package of programs was written in Matlab and the code is entirely original. Current capabilities and features of the extensive code include:

- Flexible variable-based user interface;

- Works with arbitrary three dimensional configurations of wires;

- Uses novel composite multiple domain basis functions (MDBF), enabling higher efficiency in modeling curved and lowfrequency structures; within the MDBF framework, supports piecewise linear (PWL), piecewise sinusoidal (PWS) and arbitrary shaped interpolating MDBFs.

- Supports multiple generators, as well as of plurality of lumped and distributed load types;

- Supports symmetry planes with arbitrary combination of $\mathrm{XY}, \mathrm{YZ}$ and/or $\mathrm{XZ}$ planes;

- Displays the geometry and elements of the structure graphically;

- Computes currents, as well as network parameters (admittance, impedance, and scattering matrixes $\mathbf{Y}, \mathbf{Z}, \mathbf{S}$, respectively) in one of two modes: exciting one port at a time or all ports simultaneously;

- Estimates solution errors through usage of the condition number [6];

- Computes far field radiation patterns, gain, and radiated power;

- Generates parameterized geometries for several types of antennas, such as dipole, monopole, corrugated monopole,
Input, test and process Geometry

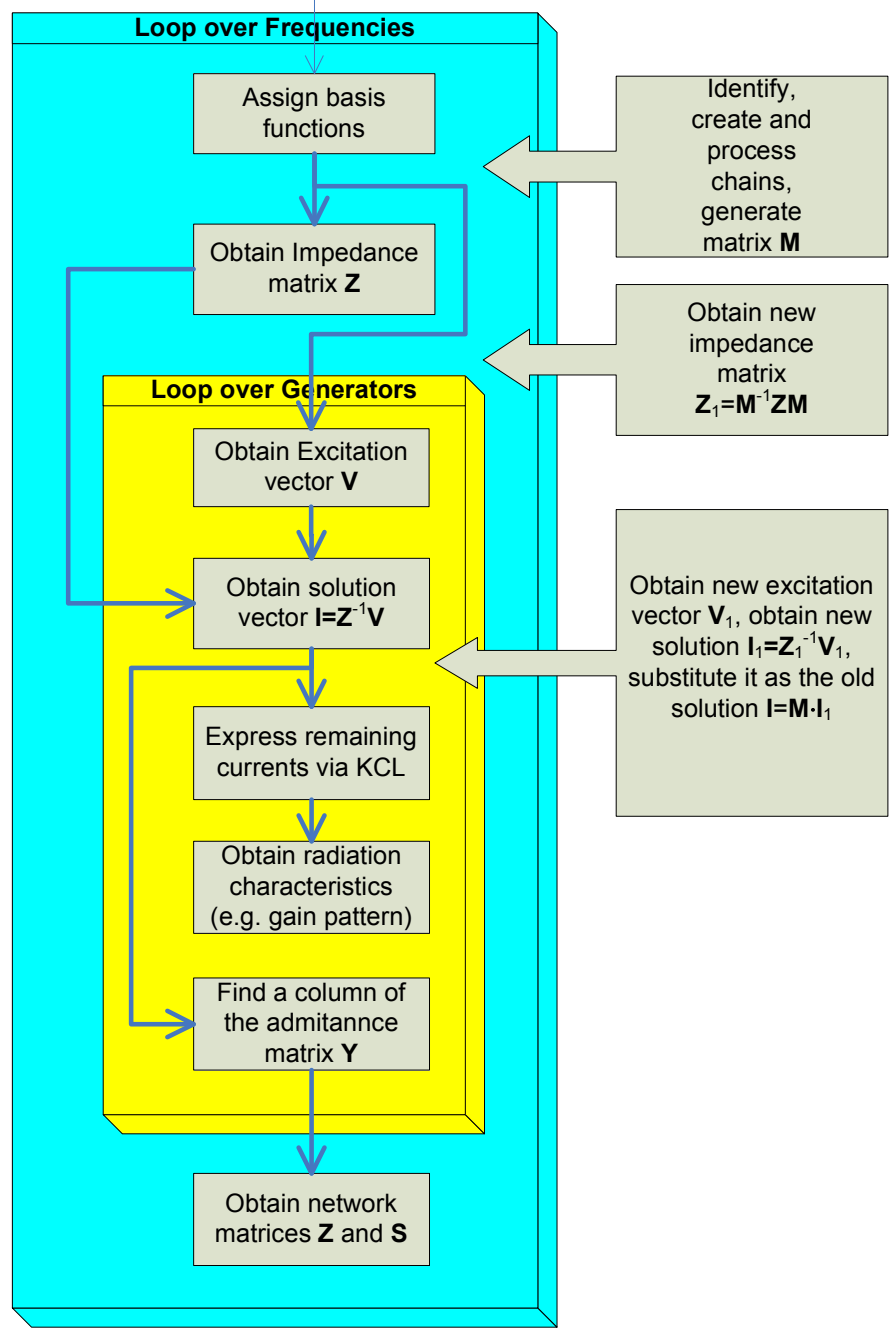

Figure 1. Flowchart of implementing MDBF. 
meandered monopole, loop, Archimedean and equiangular spiral antennas.

There is also additional functionality to co-work with two versions of WIPL-D [4] and with PCAAD [18]:

- Import and export of WIPL-D compatible files defining geometry and definitions (.IWP, SMB), current on wires and plates (.CU1), containing network parameters (.AD1), far field radiation patterns (.RA1) and near field (.NF1);

- Direct conversion between PCAAD and WIPL-D file formats for the geometry files;

- An option of executing WIPL-D software to process a project;

The code was successfully tested against many samples selected from WIPL-D reference examples as well as against some of the references from [1,21,22]. Some of the test results are provided in [6]. A sample of results showing advantages of MDBF versus standard MoM is shown in Figure 2. Moreover, the code or its parts were used in several studies and resulted in publications [6-10,12-17,19,23,24].

\section{Conclusion and Next Steps}

A new moment method code had been realized and tested on several examples. The code has an embedded automatic re-meshing mechanism and permits aggregation of the basis functions under the macrotype multiple domain basis functions of arbitrary shape with very little computational overhead. The multiple domain basis functions enable efficient modeling of curved structures, whilst requiring very little additional coding. As such it is also well suited to model smoothly bent objects using existing codes supporting only piecewise linear or piecewise sinusoidal basis functions. The studies showed that the proposed method offers improved speed compared to a standard MoM. An additional feature in the code takes advantage of the unobvious repetitions in a structure and permits to accelerate the filling in the impedance matrix, subject to the accuracy requirements. The tests have shown that for the bent objects the method produces accurate results within much shorter time than a standard MoM. The method's efficiency increases for high accuracy requirements.

The next steps in advancing the code are expected to include support for higher order basis functions [2], implementation of characteristic basis functions [25], as well as optimization of the impedance matrix routines support of quadrilateral elements $[2,4]$ and full support for a simple yet efficient pre-conditioning technique already developed by the author for the wire junctions $[19,20]$.

\section{Acknowledgments}

This work was funded in part by the Department of Electronics and Telecommunications, Norwegian University of Science and Technology, Trondheim, Norway.

\section{References}

1. C. A. Balanis, Antenna Theory: Analysis and Design, $2^{\text {nd }}$ Ed., 1997.

2. B. M. Kolundzija and A. R. Djordjevic, Electromagnetic Modeling of Composite Metallic and Dielectric Structures, Artech House, 2002.

3. List of numerical codes available at http://www.clemson.edu/ces/cvel/modeling/EMAG/SearchPage.php .

4. B. M. Kolundzija, J. S. Ognjanovic, and T. K. Sarkar, WIPL-D Microwave: Circuit and 3D EM Simulation for RF \& Microwave Applications - Software and User's Manual, Artech House, 2006, 400 pages.

5. W. C. Chew, J. Jin, E. Michielssen, and J. Song, Fast and Efficient Algorithms in Computational Electromagnetics, Artech House, 2001.

6. A. A. Lysko, On Multiple Domain Basis Functions and Their Application To Wire Radiators, Doctoral (Ph.D.) Thesis, Norwegian University of Science and Technology (NTNU), Norway, 2010, 304 p.

7. A. A. Lysko, "On Grouping Individual Wire Segments into Equivalent Wires or Chains, and Introduction of Multiple Domain Basis Functions." 2009 IEEE International Symposium on Antennas \& Propagation and USNC/URSI National Radio Science Meeting, South Carolina, USA June 1-5, 2009, 4 pages.

8. A. A. Lysko, "Using Piecewise Sinusoidal Basis Functions to Blanket Multiple Wire Segments." 2009 IEEE International Symposium on Antennas \& Propagation and USNC/URSI National Radio Science Meeting, South Carolina, USA, June 1-5, 2009, 4 pages. 
9. A. A. Lysko, "Modelling of Coil-loaded Wire Antenna Using Composite Multiple Domain Basis Functions." 2010 PIERS (Progress in Electromagnetic Research Symposium), Xian, China, Mar 2010, 5p.

10. A. A. Lysko, A Method of Operating a Computing Device to Perform Memoization, PCT Patent Application No PCT/IB2009/055650, 2009.

11. M. Donald, "Memo Functions and Machine Learning," Nature, No. 218, 1968, pp. 19-22.

12. A. A. Lysko, Cache in Computation Intensive Applications, Internal Presentation, CSIR, RSA, 2010.

13. J. Mosig, and E. Suter, "A Multilevel Divide and Conquer Approach to Moment Method Computations," in proc. of Int'l Symp on Recent Advances in Microw. Technol. (ISRAMT'99), Malaga, Spain, Dec 1999.

14. E. Suter and J. R. Mosig, "A Subdomain Multilevel Approach for the Efficient Mom Analysis of Large Planar Antennas,” Microwave and Optical Technology Letters, Vol. 26, No. 4, Aug. 2000, 270-277.

15. A. A. Lysko, "A Method of Applying Single Higher Order Polynomial Basis Function over Multiple Domains." Proc. of 2010 PIERS (Progress in Electromagnetic Research Symposium), China, Mar 2010,5p.

16. M. Taguchi, K. Yamashita, K. Tanaka, and T. Tanaka, "Analysis of Coil-Loaded Thin-Wire Antenna", IEEE AP-S International Symposium 3, 1990, 273-276.

17. A. A. Lysko, "On Calculation of Radiation Field Integrals for Higher-Order Basis Functions in Conical Thin Wire MoM Formulation." Proc. of the 6th WSEAS International Conference on Applied Electromagnetics, Wireless and Optical Communications, Trondheim, Norway, July 2008, pp. 104-109.

18. D. Pozar, PCAAD (Personal Computer Aided Antenna Design) v.6.0, Antenna Design Associates, Inc.; Web site available at http://www.antennadesignassociates.com/pcaad6.htm .

19. A. A. Lysko, "Reducing Condition Number by Appropriate Current Decomposition on a Junction with Several Wires", 2011 IEEE International Symposium on Antennas and Propagation and USNC/URSI National Radio Science Meeting, Spokane, WA, USA, July 3-8, 2011, 4 pages, accepted for publication.

20. A. A. Lysko, "Simple Preconditioning Technique: Empirical Formula for Condition Number Reduction at a Junction of Several Wires," XXX 2011), 13-20 August 2011, Istanbul, Turkey, submitted for review/in press.

21. IEEE Standard 1597.1, "IEEE Standard for Validation of Computational Electromagnetics Computer Modeling and Simulations," 2008.

22. IEEE Standard 1597.2, "Recommended Practice for Validation of Computational Electromagnetics Computer Modeling and Simulations," 2011.

23. M.J. Bembe, A. A. Lysko, T. C. Nyandeni, and W. Clark, "A Structural Parameter Based Modification of Energy Conscious ESPAR Antenna System through Optimization for WLAN's Dual Band Operability." Proc. of AfriComm 2010; ICIDC 2010), Nov 2010, Cape Town, South Africa, 5 pages.

24. A. A. Lysko, and D. L. Johnson, "A Study of Propagation Effects in a Wireless Test Bed." WSEAS Transactions on Communications, Issue 8, Vol. 7, Aug 2008. pp. 857 - 871.

25. J. Yeo, V. V. S. Prakash and R. Mittra, "Efficient Analysis of a Class of Microstrip Antennas Using the Characteristic Basis Function Method (CBFM),” Microw. Opt. Technol. Lett., Vol. 39, Dec. 2003.
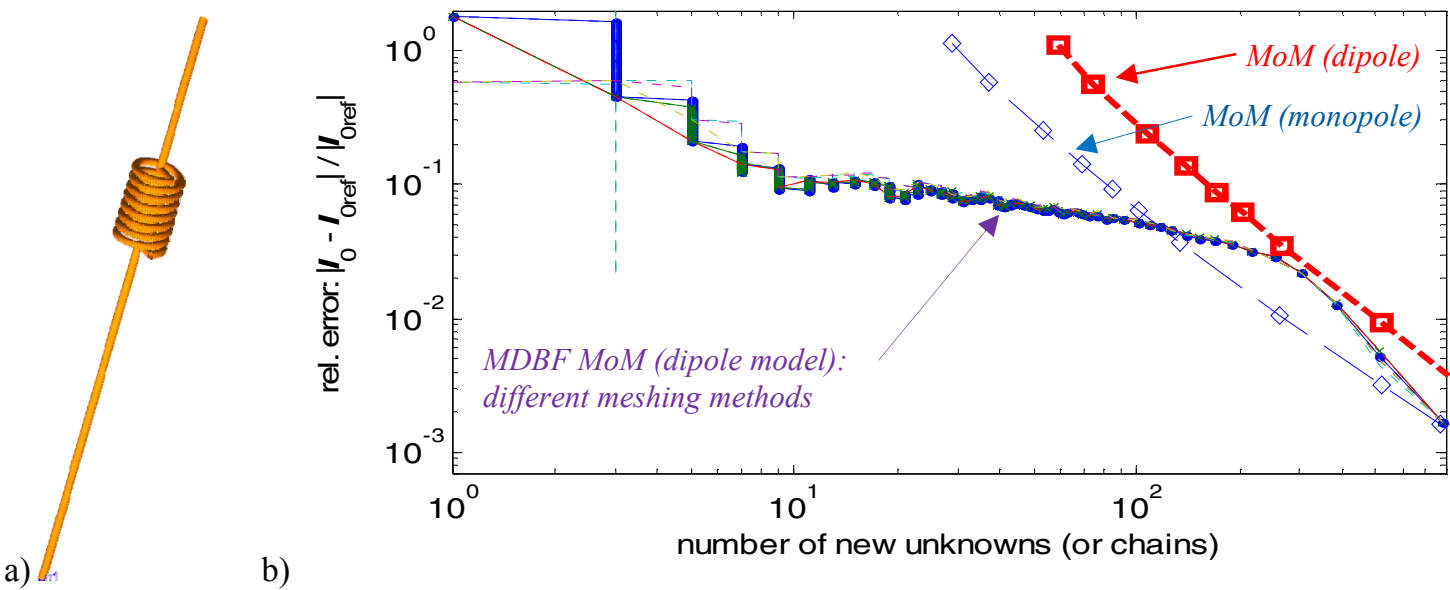

b)

Figure 2. a) Half of coil-loaded dipole model $[9,16]$; the generator is at the bottom of shown structure. b) Convergence of input impedance with growth in the number of variables (the "outwards" direction is used for the remeshing algorithm [6]). The current is compared at the feed point against a direct MoM generated reference. 